\title{
Effect of product information search type on guilt of ready meal consumption
}

\author{
Seung Cheol JUNG ${ }^{1}$, and Jaehwi KIM ${ }^{2}$ \\ (1, ${ }^{2}$ Graduate School of Chung-Ang Univ.) \\ Keywords: home meal replacement, ready meal, guilt, information search, cognitive effort, justification
}

\section{Introduction}

Demand for Home Meal Replacement (HMR; ready meal) is increasing due to the increase of dual income households and single person household. A Home Meal Replacement product is a homemade substitute product produced outside the home for the purpose of consumption in the home. It is a food prepared for convenient home meal, such as eating directly in the form of cooked or semi-cooked food. However, consumers feel a sense of guilt from ready meal consumption(Costa, Schoolmeester, Dekker, \& Jongen, 2007; Gofton, 1995; Jackson \& Viehoff, 2016; Olsen, Sijtsema, \& Hall, 2010). Comprehending previous studies dealing with feelings of guilt from ready consumption, there are two major kinds of guilt: health related guilt and effort related guilt. Health related guilt has been solved with the help of product improvement and marketing communication. However, effort related guilt is still remaining. This is because effort related guilt come from consumer's internal conflict based on their sense of obligation or duty regarding meal preparation. Thus, the purpose of this study is to reduce effort related guilt by focusing on consumer's behavior and its psychological mechanism. Specifically, the effort related guilt from ready meal consumption may vary depending on how much cognitive effort is spent in the information search process. In general, consumers want to minimize cognitive effort(Bettman, Luce, \& Payne, 1998). However, when cognitive effort gives a certain benefit to the consumers, consumers are willing to input more cognitive effort(Park, Hill, \& Raacke, 2015; Park \& Hill, 2018). The benefit, in this case, is justification of ready meal consumption. Specifically, effort related guilt of ready meal consumption can be reduced with the help of justification that appropriated amount of effort put into purchase by searching product information more deliberately.

\section{Method}

The study consists of two groups of product information search type(deliberate vs. non-deliberate between subject design), and the participants who were suitable for the ready meal were assigned randomly in two conditions. The experiment were conducted by creating an imaginary ready meal purchasing situation in a retail store. First, all participants were told brief introduction of experiment and the explanation of ready meal. And then participants read the situation statement describing the scenario of ready meal purchasing at retail store. After that, participants were manipulated in two conditions: deliberate product information search vs. non-deliberate product information search. Finally, participants reported to questionnaire which is consist of dependent variable(effort related guilt of ready meal consumption, mediator(cognitive effort justification), and control variables(e.g., NFC)

\section{Results}

As a result of manipulation check, participants in deliberate product information search condition reported that they put more cognitive effort than non-deliberate product information search condition. As a result of hypothesis testing, participants in deliberate condition report less guilt than those of non-deliberate condition (hypothesis 1 supported). In addition, as predicted, cognitive effort justification mediate the effect of hypothesis 1 (hypothesis 2 supported).

\section{Discussion}

This study is to examine the effect of the product information search type on guilt of ready meal consumption, which is a specific consumer behavior in purchasing situations, rather than the product characteristics and marketing communication. In addition, this study examine the mediation effect of cognitive effort justification. Regarding this result, companies can take advantage of cognitive effort justification based on consumer's product information search type such as showing deliberate consumer image on POP(Point of purchase). However, as discussed above, consumers tend to input less cognitive effort. So, in reality, it is difficult to make consumer search product information more deliberately in purchase stage. Nevertheless, if consumers put appropriate amount of effort in information search, it can reduce consumer's guilt from ready meal consumption.

\section{References}

Ana, I. D. A., Schoolmeester, D., Dekker, M., \& Jongen, W. M. (2007). To cook or not to cook: a means-end study of motives for choice of meal solutions. Food quality and preference, 18(1), 77-88.

Baumeister, R. F., Stillwell, A. M., \& Heatherton, T. F. (1994). Guilt: an interpersonal approach. Psychological bulletin, 115(2), 243.

Jackson, P., \& Viehoff, V. (2016). Reframing convenience food. Appetite, 98, 1-11.

Park, J., \& Hill, W. T. (2018). Exploring the role of justification and cognitive effort exertion on post-purchase regret in online shopping. Computers in Human Behavior, 83, 235-242. 